war im Versuche IV der Zuckergehalt des Pfortaderblutes 0,102 und mit Salzsäure erhitzt 0,130. Die Versuche, die freilich nicht zahlreich genug sind, würden also als Resultat ergeben, dass der Zucker des Pfortaderblutes Dextrose ist, dass ferner in einzelnen Versuchen im Blute Dextrin durch seine Umwandlung in Zucker nachzuweisen ist. Die negativen Befunde können nicht auf $\mathrm{Ab}$ wesenheit von Dextrin mit Bestimmtheit gedeutet werden, da minimale Dextrinmengen bei der Umwandlung kaum durch unsere analytischen Bebelfe nachzuweisen wären. Ein einziges positives Resultat ist darum sehr bedeutungsvoll, weil es die Möglichkeit einer solchen Resorption constatirt.

\title{
Zur Frage über das Material, aus welchem die Leber Zucker bildet.
}

Yon

\section{J. Seegen (Wien).}

Ich hatte vor einigen Jahren in einer gemeinsamen Arbeit mit Dr. Kratsehmer durch eine Reihe von Versuchen festgestellt ${ }^{1}$ ), dass in der Leber, welche einem Thiere rasch nach der Tödtung excidirt wird, der Zuckergehalt von 0,4 bis auf $3 \%$ ansteigen kann, ohne dass der Glycogengehalt eine Abnahme erleidet. Bei allen von uns untersuchten Hunden wuchs der Gesammtzucker (Zucker + Kollehydrate) in dem Maasse, als der Zucker zunahm, was unmöglich hätte geschehen können, wenn der Zuckerzunahme eine Glycogenabnahme parallel gegangen wäre. In einer zweiten Reihe von Versuchen wurde anch das Glycogen direct nach $\mathrm{Br}$ ü ck e bestimmt und bei 4 Hunden war ausnabmslos der Glycogengehalt in der ersten Stunde unverändert geblieben, während der Zuekergehalt von 0,4 auf 2,7 bei einem Thier und von 0,5 anf 1,2 beim

1) Seegen and Kratschmer, Ueber Zuckerbildung in der Leber, 'Pflüger's A rchiv XXII und XXIV. 
Zur Frage über das Material aus welchem die Leber Zucker bildet. 49

anderen Thiere angestiegen war. Bei zwei von den vier Hunden war der Glycogenbestand innerbalb der ganzen Versuchszeit von 72 Stunden gleich geblieben, während der Zucker einmal von 0,4 auf 3,3 und das zweitemal von 0,5 auf 2,8 angestiegen war. Bei Kaninchen fanden wir stets eine rasche Glycogenabnahme, die in in der ersten Zeit nach der Tödtung der Zuckerzunahme zu entsprechen schien, aber im weiteren Verlaufe weit bedeutender war als der Zuckersteigerung entsprach. Wenn man auch annehmen wollte, dass bei den Kaninchen das Glycogen für die Zuckerbildung verwendet wurde, konnten doch die vielen an Hunden gewonnenen Resultate nicht weggeläugnet werden und auf Grund derselben war die Thatsacbe festgestellt, dass die Zuckerbildung von Statten gehen könne, ohne dass der Glycogenbestand Einbusse erleidet, und es war der Sehluss berechtigt, dass die Leber im Stande sein müsse, Zucker aus anderem Material zu bilden. Es lag mir nun nahe, nach dem Materiale zu fahnden, aus welchem die Leber Zucker zu bilden im Stande sei. Ich wählte aus Gründen, die ich an anderer Stelle ${ }^{1}$ ) ausgeführt habe, Pepton zum Versuchsobjecte, und nachdem ich mich durch einige vorläufige Versuche uiberzeugt hatte, dass die Leber im Stande sei aus Pepton Zucker zu bilden, schritt ich zu den eigentlichen Versuchen ${ }^{2}$ ), die dreifacher Natur waren, nämlich: a) Fütterungsversuche, b) Injectionsversuche und c) Versuche an frisch excidirten Lebern, welche mit Pepton und arteriell erhaltenem Blute durch viele Stunden in Berührung blieben. Bei allen drei Versuchsarten war die Zuckerbildung beträchtlich erhöht und ich schloss die Mittheilung meiner Versuche mit dem Satze: "Es ist also denkbar, wenn auch lange noch nicht bewiesen, dass das Pepton das Material für die Znckerbildnng aus der Leber sei."

Chittenden und Lambert ${ }^{3}$ ) haben mit Rücksicht auf die Bedeutung der Frage, die sie nach ihrem vollen Umfange würdigen, meine Versuche nachgeprïft und zwar jene Versuche, bei welchen

I) Seegen, Die Einwirkung der Leber auf Pepton. Pflüger's Archiv Bd. XXV.

2) Seegen, Pepton als Material für Zuckerbildung in der Leber. Pflüger's Archiv XXVIII.

3) Chittenden and Lambert, The post mortem formation of sugar in the liver in the presence of peptones. Studies from the laboratory of physiological chemistry Sheff. scient. scool of Yale college. New Haven 1885. 
die Leber mit Pepton und arteriell erhaltenem Blute durch mehrere Stunden in Berïbrung blieb. Sie erhielten in allen ihren Versuchen eine Zunahme der Gesammtkohlehydrate, der Zucker war in geringem Maasse vermehrt, aber das Glyeogen hatte stets abgenommen, und wenn es ihnen auch nicht unwahrscheinlich scheint, dass die Zunahme von Kohlehydraten in irgend einer Weise auf die Gegenwart von Pepton zu beziehen ist (the real inerease of total carbohydrates is due in some manner to the presence of peptone), so kommen sie doch zu dem Schlusse, dass die Versuchsresultate nicht genügend sind, um meine Theorie iiber das Material, aus welehem die Leber Zucker bildet, anzunehmen und dass, wenn nicht weitere Beweise erbracht werden, sie daran festhalten muissen, dass der Leberzneker aus dem Leberglycogen stamme (and that without further proof to the contrary we must still adhere to the formation of liver sugar from the hepatic glycogen).

Als ich die Arbeit ron $\mathrm{Ch}$ ittenden und $\mathrm{Lambert}$ erhielt, war ich gerade damit beschäftigt, meine Versuche, ïber Zucker im Blute mit Rücksicht auf Ernäbrung" zum Abschlusse zu bringen. Diese Versuche ${ }^{1}$ ) waren, wie ich es im ersten Theile jener Arbeit darlegte, zu dem Zwecke unternommen, um zu ermitteln, "aus welchem Materiale der in der Leber gebildete und in's Blat übergeführte Zucker stammt." Ich hatte für meine Ansicht, dass Bernard's Theorie, der Leberzucker stamme aus Glycogen nicht stichbaltig sei, bereits mehrfache Beweise erbracht. Ich hatte gefunden, dass in der todten Leber der Zncker von 0,4 auf $3 \%$ anwachse ohne dass der Glycogenbestand abnimmt, ich hatte beobachtet, dass durch Pepton die Zuckerbildung gesteigert wird, ich hatte ferner gefunden, dass der Leberzucker Traubenzucker sei, während die Zuckerbildung durch Diastase, mit welcher Bernard die Zuckerbildung in der Leber identificirte, Maltose liefert, aber ich bielt alle diese Beweise für ungenügend, um eine so wichtige Frage endgiltig zu entscheiden und ich sagte mir, ${ }_{n}$ weil die Möglichkeit für die Leber erwiesen ist, aus einem Albuminate Zucker zu bereiten, ist damit noch nicht bewiesen, dass die Albuminate wirklicb das Material für diese Umsetzung abgeben. Weil das Glyoggen nicht so labil ist, wie es nach Ex-

1) Seegen, Zucker im Blute mit Rücksicht auf Ernährung. I u. II. Pflüger's Archir Bd. XXXvil and Bd. XXXIX. 
Zur Frage über das Material au welchem die Leber Zucker bildet. 51

perimenten im Laboratorium den Anschein hat, ist damit noch nicht erwiesen, dass es nicht in langsamer Umwandlung den Blutzucker liefert und wenn die Natur des Leberzuckers dagegen spricht, dass derselbe durch ein der. Diastase analog wirkendes Ferment entstanden sein könne, ist damit nur bewiesen, dass die bisherige Vorstellung über den Umwandlungsvorgang des Glycogens in Zucker eine irrige war, es ist aber nicht ausgeschlossen, dass die Umwandlung in einer anderen noch nicht gekannten Weise zu Stande komme und als Resultat Traubenzucker entstehen könne". Die Ernährungsversuche, so hoffte ich, sollen endgiltig dariber Aufschliusse geben, aus welchem Material die lebende Leber den Zucker bildet und meine Hoffnung wurde erfült.

In' acht- bis zehntägigen Hungerversuchen, wie in einer Reihe von Fettfütterangen, wo der Glycogenbestand so gering war, dass er kaum für die Zuckerbildung von 24 Stunden ausgereicht hätte, fand sich am Ende der Versuchsepoche der Zuckergehalt des Lebervenenblutes nahezu doppelt so gross, als der der Pfortader. Dasselbe war auch bei ausschliesslicher Fleischnabrang der Fall. Bei Fütterung mit Kohlehydraten war das Lebervenenblut gleichfalls beträchtlich zuckerreicher als das Pfortaderblut. Würde der Leberzucker aus dem Glycogen entstehen, könnte, da Letzteres nur aus einem Theile der eingeführten Kohlehydrate gebildet wurde, auch nicht ein Atom mehr Zucker aus der Leber ausgefiührt werden, als in Form von Kohlehydraten mit der Nabrung eingefübrt wurde. Es wurde also durch diese Ernährungsversuche bis zur Evidenz erwiesen, dass der in der Leber gebildete Zucker rom Nahrungsaucker wievon den mitder Nahrung eingefühten Kohlehydraten vollständig unabhängig ist, dass Fett und Eiweissköper das Material sind, aus welchem die Leber den Zucker bildet.

Die Hungerversuche und die Fütterungsversuche mit Fleisch, Fett und Kohlehydraten (Stärke) wurden an 33 Thieren ausgeführt. A us n a hmslos war der Zuckergehalt des Lebervenenblutes nahezu doppelt so gross, als der der Pfortader. Wenn man ferner in Betracht zieht, dass jeder Hunger- oder Fitterungsversuch 8-10 Tage dauerte, dass die Thiere im allgemeinen mindestens $10-12 \mathrm{k} 0$ schwer waren, dass es sich also in jedem Versuche um eine Ausfuhr von vielen hundert Gramm Zucker aus der Leber handelt, für welche keine andere Bedeckung als Fett oder Eiweisskörper im Thierleibe vor- 
handen waren, wird man wohl die Beweisskraft dieser Versuche für ausreichend erkiären. Ich hätte somit das Recht alle früher von mir erbrachten Beweise gegen die Zuckerbildung aus Glycogen fallen zu lassen und damit auch die Einwürfe, welche gegen die Stichbaltigkeit jener Beweise gemacht und fïr die Zuckerbildung aus Glycogen in's Treffen geführt wurden, unberücksichtigt zu lassen.

Aber ich möchte diess nicht thun. Die Frage, um die es sich hier handelt, ist eine fundamentale und für unser Verständniss des thierischen Stoffwechsels eine so bedeutungsvolle, dass sie nach allen Seiten geklärt werden muss, und dass inshesondere Forscher, die wie Chittenden und Lambert mit solchem Ernst an die Erörterung dieser Frage herangehen, den Anspruch haben, dass ihren Forschungsergebnissen zn jeder Zeit die vollste Beriicksichtignng zu Theil werde.

Chittenden und Lambert beginnen ihre Arbeit mit der Mittheilung eines meiner Versuche, mit Kalbsleber angestellt, bei welchem die Zunahme an Zucker nur sebr gering ist ,although pronounced, is not great." Dieser Versuch ist einer Anzahl von Vorversuchen entnommen, in denen ich einfach Leber-Pepton und Wasser durch längere Zeit in Berihhung gelassen hatte, und die für mich nur darum von Werth waren, weil sie mir zeigten, dass auch unter diesen Bedingungen eine Zunahme von Zucker und Gesammtlsohlehydraten stattfand.

Von jenen Versuchen, die ich als Beweis für Zuckerbildung aus Pepton mittheilte, haben Chittenden und Lambert nur einen angefüht, aber auch dieser eine beweist, dass die Zunahme von Zucker und Kohlehydraten nicht mehr unbedeutend war.

Chit ten den und La m bert entwickeln zunächst die von ihnen angewendete Methode. Bei diesen Versuchen, wo es sich darum handelt in zwei Leberstuicken den Zucker- und Glycogengehalt zu vergleichen; kommt es vor Allem darauf an, dass die Lebersticke nach diesen beiden Richtungen vollkommen erschöpft werdeu. Es ist diess sehr schwer zu erreichen und wir haben nach langen Vorversuchen eine Methode angegeben, die allein im Stande war, uns zum Ziele zu führen. Wir filtrirten das Leberdecoct durch ein Leinwandtuch, pressten erst mit der Hand, dann mit der Presse ab, kratzten den Leberfladen mit einem scharfen Löffel ab, verrieben ibn mit viel Wasser in der Reibschale, kochten die verriebene Leber mit viel Wasser und wiederholten diese Procedur viele Male. Chit- 
tenden und Lambert haben nicht diese Methode ausgefiuhrt, sie filtrirten das Leberdecoet durch einen Trichter, welcher durch absorbirende Baumwolle ausgestopft war (filtered through a funnel plugged with absorbent cotton), diese Operation wurde 4-5 Mal wiederholt. Das Gewebe wurde zeitweilig in einem passenden Mörser verrieben. Es scheint also, dass das Gewebe immer im Kochgefässe zurtickblieb und nach jedem Decantiren neues Wasser aufgegossen und lange gekocht war. Ob auch diese Methode zum Ziele führt, weiss ich nicht. Chittenden und Lambert nehmen als Beweis für die gänzliche Erschöpfung des Gewebes, wenn die Jodreaction kein Glycogen nachwies. Wir haben uns überzeugt, dass die Probe mit 95\% igem Alkohol viel empfindlicher sei, und dass diese noch Glycogen anzeigt, wenn die Jodreaction negativ ausfällt. Wir haben uns ferner überzeugt, dass wenn wir längst ausser Stande waren, Glyeogen in dem Pressrückstande der Leber zu entdecken (und nur dieser darf für diese feinen Proben benutzt werden), die Fehling'sche Lösung noch immer in eclatanter Weise Zucker nachweist und weitere Abkochungen und Auspressungen nöthig waren.

Der Zucker wurde in dem verdampften alcoholischen Extracte bestimmt, das ausgeschiedene und gewaschene Glycogen wurde in einer geschlossenen Flasche mit $2 \%$ Salzsäure auf $100^{\circ}$ erhitzt und in Zucker umgewandelt. Chittenden und Lambert bemerken, dass 17 Stunden nöthig sind, um diese Umwandlung zu vollbringen. Wir hatten uns wiederholt überzeugt, dass auch 12 Stunden zur vollständigen Umwandlung genügen. Der Unterschied ist vielleicht dadurch bedingt, dass ich nur mit $10-20 \mathrm{~cm}$ Flïssigkeit operirte, die in eine nachher zugeschmolzene Glasröhre eingeschlossen waren, während Chittenden und Lambert $200 \mathrm{ccm}$ zu einem Versuche verwendeten, die, wenn ich den Wortlaut verstehe, nicht in einem zugeschmolzenen Rohre, sondern in einer geschlossenen Flasche (a closed flask) erhitzt wurden. Uebrigens habe ich in meinen Versuchen das Glycogen direct durch Wägen bestimmt. Die Gesammtkoblehydrate bestimmten Chittenden und Lambert gleichfalls durch Umwandlung in Zucker und zwar wurden wieder $200 \mathrm{ccm}$ verwendet und durch 17 Stunden erhitzt. Chittenden und Lambert erwähnen, dass die saure Flüssigkeit stets gelb oder gelbbraun war, im Gegensatze zu meiuer Angabe, dass diese mit Säure erhitzte Flüssigkeit stets braun würde. Diese Nichtüber- 
einstimmung ist mir um so auffallender, da ich diese Dunkelbraunfärbung stets beobachtete im Decocte jenes Leberstïckes, bei welchem Pepton zugegen war, während das Decoct des andern Leberstickes eine schöne lichtgelbe Farbe hatte. Ich erwähne diese Differenzen in der Methode, weil sie vielleicht manche Differenzen in den Resultaten veranlassen. Uebrigens haben Chittend en und Lambert durch einen Versuch (IV) den Beweis erbracht, dass, wenn zwei Leberstiucke unter ganz gleichen Bedingungen verarbeitet werden; die erhaltenen Procentzahlen der einzelnen Bestandtheile kaum um 0,1 differiren.

Es folgen denn die angestellten Versuche. Es werden 13 Versuche mitgetheilt, davon sind einige zur Entscheidung weiterer Detailfragen angestellt. Versuche, die den Meinigen einigermaassen analog, sind 3 an Kaninchen, 2 an Katzen, 1 an einem Lamm, einer an einem Schafe und einer an einem Kalbe, in Summa 8, angestellt. Die Resultate sind folgende : a. In allen Versuchen ist in dem mit Pepton behandelten Leberstiucke die Summe der Gesammtkoblehydrate vermehrt, nur beim Kalbe und beim Lamm sind in beiden Leberstuicken Zucker und Kohlehydrate gleich. b. Die Zuckerbestimmung wurde in 5 Versuchen ausgeführt. Bei einem Kaninchen und bei einer Katze wurde die Znckerbestimmung durch kleine Unfälle unmöglich. In 3 Versuchen war in dem Peptonstück eine Zuckerzunabme und zwar bei 2 Kaninchen und beim Schafe, bei einer Katze und beim Lamm war der Zuckergehalt unverändert geblieben. c. Das Glycogen konnte nur bei den Kaninchen bestimmt werden. Die Lebern der Katzen, des Lammes und des Schafes waren glycogenfrei, ein Vorkommen, welches mich zumal in Bezug auf die Leber des Schafes und des Lammes sehr befremdet, die Kalbsleber enthielt $0,2 \%$ Glycogen. Bei den 2 Kaninchen, bei welchen der Zucker zugenommen hatte, war das Glycogen vermindert und zwar war bei dem Kaninchen I die Glycogenabnahme $0,75 \%$, während die Zuckerzunahme nur $0,17 \%$ betrug. Bei Kaninchen II stand die Zuckerzunahme von 0,5\% einer Glycogenabnahme von $0,6 \%$ gegeniiber. Bei Kaninchen III konnte ein Vergleich nicht angestellt werden, da der Zucker nicht bestimmt wurde. Bei den anderen Thieren kann ebenfalls keine Beziehung zwischen Zucker und Glycogen hergestellt werden, da das Glycogen feblt.

Ich lasse die Versuche von $\mathrm{Cb}$. und $\mathrm{L}$. hier übersichtlich folgen. 
Zur Frage über das Material, aus welchem die Leber Zucker bildet. 55

\begin{tabular}{|c|c|c|c|c|c|c|c|}
\hline \multirow[b]{2}{*}{ 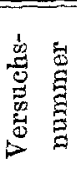 } & \multirow[b]{2}{*}{$\begin{array}{l}\text { Thier- } \\
\text { gattung }\end{array}$} & \multicolumn{3}{|c|}{ Ohne Pepton } & \multicolumn{3}{|c|}{ Mit Pepton } \\
\hline & & GIycogen & Zucker & $\begin{array}{c}\text { Gesammt- } \\
\text { kohle- } \\
\text { hydrate }\end{array}$ & Glycogen & Zucker & $\begin{array}{l}\text { Gesammt- } \\
\text { kohle- } \\
\text { hydrate }\end{array}$ \\
\hline I & Kaninchen & 6.21 & 2.74 & 10.75 & 5.46 & 2.91 & 11.08 \\
\hline II & " & 8.09 & 2.75 & 13.55 & 7.46 & 3.26 & 14.15 \\
\hline III & $"$ & 1.54 & 2.86 & 5.84 & 1.65 & verloren & 6.42 \\
\hline IX. & Katze & 0 & 1.67 & 1.89 & 0 & 1.74 & 2.13 \\
\hline $\mathrm{X}$ & 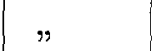 & 0 & verloren & 2.20 & 0 & verloren & 2.72 \\
\hline $\mathrm{XI}$ & Lamm & 0 & 2.51 & 2.46 & 0 & 2.52 & 2.41 \\
\hline XII & Schaf & 0 & 0.84 & 0.96 & 0 & 1.10 & 1.53 \\
\hline XIII & Kalb. & 0.22 & 2.52 & 2.87 & 0 & 2.39 & 2.86 \\
\hline
\end{tabular}

Die andern Versuche, alle an Kaninchen, wurden wie erwähnt, zur Lösung nebengehender Fragen angestellt. Versuch IV sollte beweisen, dass unter gleichen Versuchsbedingungen in beiden Stücken gleiche Resultate zum Vorschein kommen. Versuch V und VI sollten zeigen, welchen Einfluss Pepton ohne Anwesenheit von Blut übe. Die beiden Versuche ergaben gleichfalls eine bedeutende Vermehrung der Gesammtkohlehydrate bei dem mit Pepton in Berïhrung gestandenen Leberstïck. Meine Vorversuche hatten mich schon gelehrt, dass die Leber auf Kosten von Pepton auch ohne die Einwirkung des Blutes die Bildung von Kohlehydraten steigere. Dass das Blut nicht nöthig sei, beweist ja überhaupt die ganze postmortale Zuckerbildang. Die Leber bildet Zucker, solange die Zelle noch ihr Eigenleben hat. Das Zusammenbringen mit arteriell erhaltenem Blute sollte eben nur die Lebensfähigkeit der Zelle erhalten. Dass das Blut als solches weder eine Vermehrung von Zucker noch eine solche von Koblehydraten bewirkt, habe ich in einem Versuche gezeigt, dessen Resultate so eclatant sind, dass ich dieselben hier folgen lasse. Es werden 3 gleich grosse Leberstücke desselben Hundes zum Versuche genommen, a. mit Wasser ohne Pepton, b. mit Blut obne Pepton, c. mit Blut und Pepton beschickt und die beiden letzten b. und c. mit Aspiratoren verbunden; ich erbielt folgendes Resultat: 


\begin{tabular}{c|c|c|c}
\hline $\begin{array}{c}\text { Versuchs- } \\
\text { stück }\end{array}$ & $\begin{array}{c}\text { Art der } \\
\text { Behandlung }\end{array}$ & $\begin{array}{c}\text { Leberzucker } \\
\text { in \% }\end{array}$ & $\begin{array}{c}\text { Gesammtkohle- } \\
\text { hydrate }\end{array}$ \\
\hline a & mit Wasser & 2.94 & 5.0 \\
b & mit Blut & 2.94 & 5.0 \\
o & Blut u. Pepton & 3.90 & 6.6
\end{tabular}

Ch. und L. baben in Versuch VII 2 Stiicke Leber eines Kaninchens, eines mit Wasser und eines mit Blut, ohne Pepton behandelt. Die Gesammtkohlehydrate waren in beiden Stitcken gleich, aber in dem mit Blut behandelten Stiicke war ein Zuckerplus von $+0,6$ und die Glycogenabnabme betrug $-1,26$.

Ich möchte nun noch die Resultate meiner an Hunden angestellten Versuche denen von $\mathrm{Ch}$. und $\mathrm{L}$. entgegenstellen. Die Glycogenbestimmung geschah direct durch Fällung mit absolutem Alkohol und Wägung des trockenen Niederschlages.

\begin{tabular}{l|c|c|c||c|c|c}
\hline Versuchs. & \multicolumn{3}{|c|}{ Ohne Pepton } & \multicolumn{2}{|c}{ Mit Pepton } \\
\cline { 2 - 4 } & $\begin{array}{c}\text { Zucker } \\
\text { in } \%\end{array}$ & Glycogen & $\begin{array}{c}\text { Gesammt- } \\
\text { kohle- } \\
\text { bydrate }\end{array}$ & Zucker & Glycogen & $\begin{array}{c}\text { Gesammt- } \\
\text { kohle- } \\
\text { hydrate }\end{array}$ \\
\hline XXIV & 2.5 & - & 3.38 & 3.57 & - & 4.80 \\
XXV & 2.3 & - & 3.50 & 3.90 & - & 5.00 \\
XXVI & 2.1 & 2.05 & 5.20 & 2.50 & 2.08 & 7.10 \\
XXVII & 3.0 & 2.12 & 6.90 & 3.87 & 2.02 & 8.40 \\
XXVIII & 2.9 & - & 5.00 & 3.90 & - & 6.60
\end{tabular}

Die von Ch. und L. erzielten Resultate stimmen in einem Punkte mit den Meinigen überein und zwar darin, dass bei Gegenwart von Pepton die Gesammtkohlehydrate vermehrt sind. Ch. und L. weisen nach, dass die Vermehrung, wenn auch nicht sehr bedeutend, doch weit jenseits der Fehlergrenze gelegen ist und dass sie ausnahmslos in der Gegenwart von Pepton nachzuweisen ist. Die Zuckersteigerung ist in den Versuchen von $\mathrm{Ch}$. und $\mathrm{L}$. 
nur eine geringe und in den wenigen (zwei) Versuchen, in welchen auch Glycogen nebst Zucker bestimmt warde, ist eine Glycogenabnahme constatirt, die aber bei weitem die Zuckerzunabme übersteigt. Der Sehluss, zu welchem nun Ch. und L. gelangen, ist nun folgender. ,Während unsere Versuche die Angaben von S. und K. (hier ist ein Irrthum, für die Versuche mit Pepton habe ich allein einzustehen) in Bezug auf die Zunahme von Gesammtkohlehydraten bekräftigen, können wir die mässige Zunahme nicht für genügend halten, um in einer so wichtigen Frage zur Entscheidung zu gelangen. Da ferner der Zucker nur sehr wenig zunimmt und diese Zunahme nur bei gleichzeitiger Abnahme von Glycogen zu constatiren ist, liegt darin ein Grund mehr, um der Zunahme von Kohlehydraten nicht so viel Gewicht beizulegen, als dies sonst geschehen wäre." (As might otherwise be done.)

Ch. und L. erwähnen, dass ihre Resultate, die Zuckerbildung gehe mit der Abnahme von Glycogen einher, in Uebereinstimmung sind mit denen, welche Boehm und Hoffmann, und früher Delprat gefunden haben. Sie führen an, dass die Erstgenannten unsere Methode der Glycogenbestimmung nicht frei von analytischen Fehlern gefunden haben. $\mathrm{Cb}$. und L. vergessen hinzuzufügen, dass wir diese gegen unsere ersten Mittheilungen ,über Zuckerbildung in der Leber" gerichteten Einwürfe in einer 2. Arbeit ausfübrlich widerlegt haben, dass wir den berechtigten Anforderungen von Boehm und Hoffmann rolle Rechnung getragen und in einer Reihe nener Versuche das Glycogen direct bestimmt haben und dass auch bei den neuen an Hunden gemachten Versuchen der Zucker anwuchs von 0,5 bis auf $3 \%$, während das gewo gene Glycogen stets gleich blieb. Wir glaubten wohl damit den Einwürfen von Boehm und Hoffmann gerecht geworden zu sein, und es fördert wohl kaum eine Frage, wenn durch ernste Arbeit widerlegte Einwürfe wie die Geister der Dahingeschiedenen aber und abermals in den Kampf geführt werden.

Auf Delprat's Versuche habe ich nicht geantwortet. Dieselben erschienen in holländischer Sprache, die ich nicht verstehe, eine Uehersetzung in's Deutsche, die ich durch gütige Vermittlung von Prof. Stokvis anfertigen liess, war nicht gelungen, ich lernte die Arbeit aus dem Referate ${ }^{1}$ ) kennen und ich fand in demselben,

1) Maly's Jahresbericht 1881, S. 321. 
dass, abgesehen von den Versuchsergebnissen, auch die gleichen von uns angewandten Methoden zu verschiedenen Ergebnissen führten. So z. B. verlief in Delprat's Versuchen bei der Zuckerbestimmung die Reduction nie mit Bildung von rothem Kupferoxydul und die Bestimmung war nur bis auf $1 / 2 \mathrm{ccm}$ der zuckerhaltigen Fliissigkeit genau, ferner bei Bestimmung des Gesammtzuckers wurde das Kupferoxydul zumeist in Lösung gehalten und war die Bestimmung bis auf $1-2$ cem ungenau. Ferner differirten die directe und die indirecte Glyeogenbestimmung um kolossale Grössen, es wurde z. B. durch Wägung erhalten 10,9 und dureh indirecte Bestimmung 5,0 gr Glycogen. Alle diese auf Ungenauigkeiten der Methoden hinzielenden Erfahrungen waren mir ganz neu, ich hatte sie weder in meinen mit Kratschmer gemeinsam ansgeführten Arbeiten, noch in den unzähligen Versuchen, die ich selbst ausgefubrt habe, zu machen Gelegenheit. Ich konnte an der Verlässlichkeit der Methoden nicht zweifeln, hatte aber auch nicht das Recht, das Können Delp rat's zu discutiren und musste darum seine Einwürfe unerwidert lassen. Nicht besonders ermuthigend für die Discussion der Del prat'schen Versucbe war der Kaninchenversuch 3, bei welchem die Leber nur Spuren von Zucker enthält, ein Vorkommen, welches mir bei meinen zahllosen Versuchen nie begegnet ist. Delprat fand dieses merkwïrdige Vorkommen auch noch ein zweites Mal bei einer Katze. Wenn ich übrigens Delprat's Versuche verwerthen wollte, wären sie eine Bestätigung für meine Angaben. Bei dem einzigen Hundeversuche, den er anführte, stieg der Leberzucker von 0,3 auf $1,4 \%$ und die directe Glycogenbestimmung zeigte nicht bloss eine Abnahme, sondern eine Vermehrung des Glycogens um 1\%!

Wenn Ch. and L. ihre Versuchsresultate ohne Voreingenommenheit analysirten, wiirden sie finden, dass durch die ausnahmslose Zunahme der Gesammtkohlehydrate bei der mit Pepton digerirten Leber die Cardinalfrage, auf die es ankommt, experimentell entschieden ist, dass nämlich die Leber im Stande ist au s Eiweisskörpern Kohlebydrate zu bilden. Es ist ganz gleichgültig, ob grosse oder kleine Mengen Kohlehydrate neu gebildet wurden, die Menge hängt wabrscheinlich von der Leistungsfähigkeit der Leberzellen ab, und dass diese nicht bei allen Thierarten und Thierindividuen gleich ist, wird dadurch bewiesen, dass ich in meinen Versuchen an Hunden eine weit grössere Zu- 
nahme von Kohlehydraten nachgewiesen habe. Mit der Verschiedenheit der Leistungsfähigkeit hängt es wohl anch zusammen, dass bei Ch. und L. Versuchsthieren nur die Gesammtkohlehydrate in beträchtlicher Menge vermehrt waren, ohne dass die Zuckerbildung wesentlich gesteigert war, d. h., dass als Spaltungsprodukt aus den Eiweisskörpern sich Dextrine gebildet hatten, die nur zum kleinsten Theile in Zucker umgewandelt waren. Der Umstand, dass der Zuckerzunahme eine Glyeogenabnahme entspricht, sollte Ch. und L. nicht beirren; schon die Thatsache, dass die Glycogenabnahme weit grösser ist als die Znckerzunahme, beweist, dass diese zwei Prozesse nicht zusammenhängen und ihre getrennten Wege gehen. Ich habe überdiess in zahlreichen Versuchen bewiesen, dass bei Kaninchen eine sehr rasche Abnahme des Glycogens eintritt, dass aber bei anderen Thieren, z. B. bei Funden, der Zucker anwächst, ohne dass der Glycogenbestand sich ändert. Ich begreife es vollkommen, dass es $\mathrm{Ch}$. und $\mathrm{L}$. wie vielen Andern schwer wird, sich von der eingewurzelten Idee der Zuckerbildung aus Kohlehydraten loszumachen und dass sie darum meiner Lehre Misstrauen entgegenbringen. Bernard sagte an einer Stelle seiner Arbeiten, in welchen er sich gegen die zahlreichen Gegner seiner Entdeckung, dass die Leber Zucker bildet, wendet, „man dürfe doch von loyalen Gegnern erwarten, dass sie die Experimente, die man als Beweismaterial erbracht hat, in ganz gleicher Weise ausführen." Es wäre gewiss in Bernard's Sinne gehandelt, wenn die Vertheidiger seiner Anschaung uiber das "Wie" der Zuckerbildung, welche meine Versuche controliren, dieselben genau so ausführten, wie ich es angegeben, und z. B. statt der Kaninchen Hunde als Versuchsobjecte wählten. Bei den Ersteren wird die Labilität des Glycogens stets einen beirrenden Einfluss ïben, der bei den Versuchen an Hunden wegfällt. Dann dürften die Widersprïche, die noch heute in den von $\mathrm{Ch}$. und L. und den von mir gefundenen Resultaten bestehen, hoffentlich schwinden.

Ich möchte nun noch einen zweiten Punkt besprechen, in Bezug auf welchem Ch. und L. mit mir im entschiedensten Gegensatze stehen, nämlich die Natur des. Leberzuckers. Hier handelt es sich um eine vollständige Missdeutung eines von den genannten Forschern gefundenen Intersuchungsresultates. Ich habe zuerst allein und dann gemeinschaftlich mit $\mathrm{Kratschmer}$ eingehende Untersuchungen tiber die Natur des Leberzuckers gemacht. Die ersten von mir 
ausgeführten Versuche ${ }^{1}$ ) wurden mit dem Dialysate von ausgepresster Kalbsleber gemacht, dasselbe wurde eingeengt mit absolutem Alcohol behandelt und der Zucker als Zuckerkali gefällt. Dieses (welches schon das characteristische firnissartige Aussehen von Traubenzuckerkali hat) wurde gelöst und der Zuckergehalt durch Gährung und Reduction bestimmt und die Ablenkungsgrösse festgestellt. Alle gewonnenen Ziffern stimmten vollkommen für Traubenzucker. Nachdem Musculus und v. Mering mitgetheilt hatten, dass es ihnen $2 \mathrm{Mal}$ gelnngen sei, in der todtenstarren Hundeleber neben Traubenzucker auch Maltose nachzuweisen, gingen wir an die erneute Untersuchung dieses Gegenstandes ${ }^{2}$ ). Da Musculus und v. Mering die Methode, durch welche ihnen der Nachweis von Maltose in der Leber gelungen ist, nicht angeben und wir annehmen mussten, dass sie die fractionirte Fällung durch Aether, den sie für die durch Fermente umgewandelte Kohlehydrate benutzt haben, angewendet haben, benutzten auch wir diese Methode. Leberdecocte wie kalte Leberextracte wurden durch fractionnirte Fällung behandelt. Wir erhielten Resultate, die entweder mit Bestimmtheit zeigten, dass nur Traubenzucker vorbanden sei - es stimmten nämlich Gährungs- und Reductionsresultat vollkommen und die Ablenkungsgrösse war die des Traubenzuckers - oder es war bei andern Lebern in die Fällungs rückstände auch zugleich etwas Dextrin mitgegangen. Wir haben aber ferner, nachdem wir uns durch Vorversuche tiberzeugt hatten, dass Maltose rasch dialysire, während Dextrin nur sehr langsam in's Dialysat übergehe, eine grosse Reihe von Versuchen mit dem Dialysat von Leberextracten direct ausgeführt. Durch Gährung und durch Reduction wurden die Zuckermengen bestimmt, ein Theil des Dialysats warde mit Salzsäure in der geschlossenen Röbre durch 24 Stunden in kochendem Wasserbade erhitzt und dann in der Flüssigkeit der Röhre der Zuckergehalt bestimmt, es wurde ferner im Polarisationsapparate die Ablenkungsgrösse gemessen. Alle diese Factoren stimmten rollkommen für Traubenzucker. Ch, und L., die von meinen Beweisen nur den anführen, dass ich aus dem Leberextracte das dem Traubenzucker entsprechende Zuckerkali dargestellt habe, sind auf Grundlage

1) Pflüger's Archiv Bd. XIX.

2) Pflüger's Archiv Bd. XXII. 
ibrer Versuche zu dem Schlusse gelangt, dass der Leberzucker ein Gemenge von Dextrose und Maltose sei. Der Beweis, den sie dafür erbringen, ist folgender: sie fanden, dass die Gesammitmenge der Kohlehydrate, welche durch Ueberführen des Leberdecoctes in Zucker mittelst Säure gewonnen wurde, stets grösser war als die Summe von Zucker und Glycogen (diese als Zucker berechnet), dass aher die ziffermässige Uebereinstimmung der Gesammtkohlehydrate mit Glycogen und Zucker eine vollständige wird, wenn die Zuckerlösung, ehe man deren Quantität bestimmt, durch 17 Stunden mit Säure erhitzt wurde. Durch das Erhitzen, so meinen Ch. und L., ist Maltose in Dextrose verwandelt worden. Wäre es nicht eben so gut denkbar, anzunebmen, dass in der den Zucker enthaltenden alcoholischen Lösung auch Dextrin mit vorbanden war, welches in der Röhre in Zucker umgewandelt wurde. Ch. und L. fiuhrten ihre Zuckerbestimmung so aus, dass $200 \mathrm{ccm}$ des Leberdecocts auf eine kleine Menge eingedampft und mit einer grossen Menge Alcohol behandelt wurden, das alcoholische Filtrat abgedampft, in Wasser aufgenommen und der Zucker bestimmt wurde.

$\mathrm{Ch}$. und L. geben nicht an, was für Alcohol sie benutzt und in welchem Verhältnisse zur Zuckerlösung. Dem Einwande, dass das Zuckerplus in der mit Säure erbitzten Zuckerlösung Dextrin sein könnte, suchen sie dadurch zu begegnen, dass sie anführen, sie hätten mit dieser Lösung Gährungsversuche gemacht und es sei alles vollkommen vergohren, ferner dass wenn man nach Dextrin suchen wollte, wäre es natürlich nach demselben im Glycogenniederschlag auszuschanen, it would of course be more natural in seeking for dextrin to look in the glycogen praecipitate." In diesen beiden Punkten sind Ch. und L. im Irrthum. Ich und Kratsehmer haben in jener Arbeit, durch welche wir auf die Einwürfe von Boebm und Hoffmann antworteten ${ }^{\mathbf{1}}$ ), Versuche, an Meerschweinchen ausgeführt, mitgetheilt, bei welchen es sich berausstellte, dass bei Fällung des Leberdecocts mit 90\% igem Alcohol die gefundene Glycogenmenge $6,9 \%$ betrug, während das Präcipitat, welches bei Fällung mit absolutem Alcohol entstand, 7,4\% betrug. Bei der friiheren Fällung mit $90 \%$ war also ein Kohlehydrat, wahrscheinlich Dextrin, in der alcoholischen Zuckerlösung

1) Pflüger's Archiv Bd, XXIV, S. 476. 
zurïckgeblieben. Wir haben auch durch directe Versuche, die schon vọn Muskulus und Barfoed beobachtete Gährungsfähigkeit des Dextrins nachgewiesen, insbesondere dann, wenn das Dextrin sich in Zuckerlösung befindet. Es ist also wohl mehr als wahrscheinlich, dass die Differenz im Zuckergehalte vor und nach dem Erhitzen der Lösung mit Säure auf Rechnung von Dextrin zu beziehen ist. Wäre diess durch absoluten Alcohol gefällt worden, oder hätten sie die Lösung dialysirt, dann wïrden Ch. und L. dieselbe Erfahrung gemacht, welche 0 . Nasse ${ }^{1}$ ) und wir gemacht haben, dass die Erhitzang mit Säure das Reductionsvermögen der Zuckerlösung nicht zu ändern im Stande ist, dass also kein Grund vorliegt, den Leberzucker als ein Gemenge von Maltose und Dextrose anzusehen.

\section{Ueber Sehstörungen nach Operationen im Bereich des Vorderhirns.}

Von

\section{Prof Sigm. Exmer und Dr. Josef Paneth.}

Die Frage, ob blos Exstirpationen im Bereich der von $\mathrm{Munk}$ abgegrenzten Sehsphäre, oder auch an anderen Theilen des Hundehirns, insbesondere am Vorderhirn, Sehstörungen nach sich ziehen, steht im Vordergrunde der Discussion, die sich an die Localisation der Functionen im Gehirn knuipft. Dieser Umstand, der ausser in der Literatur auch noch in den Verhandlungen der physiologischen Section der diesjährigen Naturforscher-Versammlung zu Tage trat, rechtfertigt es, wenn wir nachstehend iiber unsere einschlägigen Erfahrungen kurz berichten, die wir im Verlaufe einer nach ganz anderen Zielen gerichteten Untersuchung zu machen Gelegenheit hatten.

Wir haben an sechs Hunden deu Gyrus sigmoideus der einen Seite oder theilweise exstirpirt, und in fünf dieser Fälle Sehstörungen beobachtet, welche bis zu vier Wochen anhielten. In keinem war

1) O. Nasse, Bemerkungen zur Physiolopie der Kohlehydrate. Pflügers Archiv. Bd. XIV. 